REVISTA DE DERECHO UNED, NÚM. 21, 2017

\title{
PREVISIÓN ANTITERRORISTA PARA LA REGLAMENTACIÓN FUTURA DE LOS DRONES
}

\section{THE FUTURE LEGALITY OF DRONES ABOUT THE TERRORIST FIGHT}

\author{
Victoriano Perruca Albadalejo \\ Doctor en Derecho y Ciencias Sociales por la UNED
}

Resumen: Es el propósito de este estudio proponer un reglamento español sobre el régimen jurídico de los drones de pequeño tamaño con funciones de vigilancia y de seguridad en contextos tanto civiles como militares, previendo al respecto los lugares donde tales actividades puedan tener lugar, sus requisitos aeronáuticos y de responsabilidad civil derivada como consecuencia de tales vuelos no tripulados. Todo ello a la luz de una perspectiva propia de una eficaz lucha antiterrorista.

Palabras clave: drones, vuelos no tripulados, responsabilidad, antiterrorismo.

Abstract: In this study we are doing a spanish legal act about some little drones which they have security and vigilancy functions as in civil context as in military context.

We can see the features of places when they could work and other requires as the civil responsability of these special flyes according to get a good antiterrorisim fight.

Keywords: drones, fliy without pilot, responsability, fight against the terrorism.

Recepción original: 10/09/2017

Aceptación original: 27/10/2017 
Sumario: I. Introducción: objeto y sus premisas europarlamentarias. II. Estado actual de la cuestión. 1. Requisitos locativos. Lugares donde la actividad puede tener lugar. 2. Requisitos de la aeronave y ambientales. La grabación por vigilancia a través del dron. 3. Requisitos personales de pilotaje: Registro de AESA (comunicación y autorización). 4. Requisitos de la empresa operadora propietaria y responsable de los drones. 5. Requisitos de vuelo. III. Conclusión: propuesta propia de un reglamento para España. Exposición de Motivos. Su objetivo y necesidad de cobertura. Su nervio principal y peligro que se deriva del objetivo anterior. La normativa internacional de referencia y la contribución española. La definición española de «vehículo aéreo no tripulado" y sus requisitos. Su matriculación. Su circulación aérea. Sobrevuelo de otros Estados y régimen aeroportuario. La Responsabilidad por daños e investigación de accidentes graves.

\section{INTRODUCCIÓN: OBJETO Y SUS PREMISAS EUROPARLAMENTARIAS}

Este estudio, habida cuenta el vacío de la normativa ${ }^{1}$ que en esta materia se constata, tiene por objeto proponer un Reglamento aeronáutico sobre el régimen jurídico futuro de esta clase de ingenios espaciales no tripulados.

Para ello se parte de dos postulados formulados en el Reglamento del Parlamento europeo núm. 216/2008 en sus consideraciones 5 y 29, según los cuales no resulta adecuado someter a todas las aeronaves a normas comunes pero sí proporcionadas al nivel de seguridad requerida tanto para la aviación civil en general como para la protección del medio ambiente. Para alcanzar tales objetivos tienen un papel preponderante los Estados miembros de la Unión Europea (UE) y ésta otro ya que lo es sólo subsidiario.

${ }^{1}$ NORMATIVA BÁSICA: A) Internacional: Convenio sobre Aviación Civil Internacional firmado en Chicago el 07.12.1944. Texto Constitutivo de la UE: art. 251. Reglamento (CE) núm. 216/2008 del Parlamento Europeo y del Consejo de 20.02.2008 sobre normas comunes en el ámbito de la aviación civil y por el que se crea una Agencia Europea de seguridad Aérea. (DOUE de 19.03.2008). B) Nacional: Constitución española. Ley 48/1960, de 21 de Julio, de navegación aérea. Ley 39/1992, de 26 de noviembre, de RJAPAC, en lo que rija, y la actualmente vigente 39/2015 que la sustituyó. Ley 21/2003, de 7 de julio, de seguridad aérea. Real Decreto-Ley 12/1978, de 27 de abril, sobre fijación y delimitación de facultades entre los Ministerios de defensa y de Fomento (actual). Real Decreto 660/2001,de 22 de junio, por el que se regula la certificación de las aeronaves civiles y de los productos y piezas relacionadas con ellas. Real Decreto 2218/2004, de 26 de noviembre, por el que se aprueba el Reglamento de aeronavegabilidad de la Defensa. Orden de Presidencia del Gobierno de 14 de marzo de 1957 


\section{ESTADO ACTUAL DE LA CUESTIÓN}

Por tanto, los puntos que se tratan expresamente aquí son los siguientes en función del estado actual de la cuestión:

\section{Requisitos locativos. Lugares donde la actividad puede tener lugar}

Actualmente se pueden utilizar drones civiles para la realización de trabajos aéreos, entre ellos la filmación y grabación.

No obstante, en un primer momento, y hasta que no esté aprobada la reglamentación definitiva que dispone la Disposición final segunda de habilitación normativa, en su segundo apartado, del Real Decreto Ley 8/2014, de 4 de julio, de aprobación de medidas urgentes para el crecimiento, la competitividad y la eficacia, las operaciones que se pueden realizar se limitan a zonas no pobladas y al espacio aéreo no controlado. En caso de recintos interiores, la actividad de grabación de vigilancia también puede ser nocturna, no sólo diurna.

\section{Requisitos de la aeronave y ambientales. La grabación por vigilancia a través del dron}

La normativa permite para una aeronave de hasta $25 \mathrm{~kg}$, grabar en exteriores, pero ha de hacerse de día y en condiciones meteorológicas visuales, en zonas fuera de aglomeraciones de edificios en ciudades, pueblos o lugares habitados o de reuniones de personas al aire libre, en espacio aéreo no controlado, dentro del alcance visual del piloto, a una distancia de éste no mayor de $500 \mathrm{~m}$. y a una altura sobre el terreno no mayor de 400 pies (es decir, como máximo $120 \mathrm{~m}$. sobre el terreno).

\section{Requisitos personales de pilotaje: Registro de AESA (comunicación y autorización)}

Para conseguir la habilitación como operador de drones civiles para realizar este tipo de trabajos el régimen establecido es de comunicación previa (que no de autorización) y declaración responsable, por lo que es necesario tan solo un acuse de recibo una vez que presente en el Registro de la Agencia Estatal de Seguridad Aérea 
(AESA) la declaración responsable junto con la documentación exigida, cuyo acuse de recibo le habilitará como operador de drones civiles.

En todo caso para la realización de fotografías o filmaciones con cualquier tipo de aeronaves, tripuladas o no, es necesario obtener una autorización específica de AESA (Orden de Presidencia del Gobierno de 14 de marzo de 1957), en cuya página web figura la información e instrucciones al respecto, en concreto el Procedimiento para la obtención de permisos de vuelo de trabajos aéreos.

Todos los pilotos de drones, indistintamente del tamaño de la aeronave, deberán acreditar una serie de requisitos:

A) En primer lugar, acreditar que posee los conocimientos teóricos necesarios para obtener una licencia de piloto. Se puede hacer de tres formas:

- tener o haber tenido (en los últimos 5 años) una licencia de piloto (cualquier licencia), pudiendo incluirse la de planeador, de globo o de ultraligero;

- demostrar de forma fehaciente que disponen de los conocimientos teóricos para obtenerla (por medio certificado de conocimientos teóricos emitido por una organización de formación aprobada por AESA, ATO, o, en el caso de que esos conocimientos correspondan a una licencia de piloto de ultraligero, mediante un certificado individual como APTO tras realizar el correspondiente examen oficial de conocimientos teóricos),

- o si el peso máximo al despegue no es superior a $25 \mathrm{~kg}$ por medio de un certificado básico o avanzado emitido por una organización de formación aprobada (ATO) tras superar un curso al efecto. Esta última podría ser en el caso del Proyecto.

La lista de organizaciones de formación aprobadas puede encontrarse en la página web de AESA.

Además, si no tuvieran una licencia de piloto, deben acreditar que tienen más de 18 años.

B) En segundo lugar, por su peso deberán presentar un determinado certificado médico, el de Clase LAPL (para aeronaves de hasta $25 \mathrm{~kg})$.

C) Finalmente deberán acreditar que disponen de los conocimientos adecuados de la aeronave que van a pilotar y de su pilotaje, por medio de un documento que puede ser emitido por el operador, por el 
fabricante de la aeronave o una organización autorizada por éste, o por una organización de formación aprobada.

\section{Requisitos de la empresa operadora propietaria y responsable de los drones}

Indistintamente del tamaño de la aeronave tripulada por control remoto, todos los operadores de drones deberán cumplir los siguientes requisitos:

a) Disponer de la documentación sobre la caracterización de la aeronave (configuración, prestaciones).

b) Contar con un Manual de operaciones en el que establezca los procedimientos de la operación (por ejemplo, criterios para designar las zonas de despegue y aterrizaje, de condiciones meteorológicas para poder volar, etc.).

c) Haber realizado un estudio aeronáutico de seguridad de la operación.

d) Establecer un programa de mantenimiento de la aeronave.

e) Tener un piloto que cumple los requisitos establecidos.

f) Disponer de un seguro conforme a la normativa vigente.

g) Adoptar las medidas adecuadas para que la aeronave no sufra actos de interferencia ilícita durante las operaciones, incluyendo interferencias deliberadas al enlace de radio y acceso no autorizado a la estación de control, durante el vuelo, o a la ubicación donde se almacene la aeronave.

h) Garantizar que la operación se realice a una distancia mínima de 8 kilómetros de cualquier aeropuerto o aeródromo, o de 15 si en él se puede operar en vuelo instrumental, o caso contrario haberse puesto de acuerdo con el mismo. Sin embargo, en el caso de operadores de aeronaves de hasta 25 kilos de peso máximo al despegue no necesitan solicitar una autorización para poder operar sino que únicamente tienen que presentar en AESA una comunicación previa y declaración responsable conforme su aeronave cumple todas esas exigencias, junto con la documentación que lo acredite:

- Esa comunicación previa y declaración responsable habrán de presentarse a AESA con una antelación mínima de 5 días antes del inicio previsto de la operación. 
- AESA facilitará al interesado un acuse de recibo que servirá para acreditar ante terceros que el interesado está legalmente habilitado para realizar esa actividad.

- Cualquier modificación que se produzca también deberá ser notificada a AESA con una antelación mínima de 5 días, presentando la documentación original que haya sido modificada junto con declaración responsable que cubra las modificaciones introducidas.

\section{Requisitos de vuelo}

Todos los drones, sin excepción, deben de llevar fijada en su estructura una placa de identificación en la que deberá constar, de forma legible y a simple vista, la identificación de la aeronave, mediante la designación específica, número de serie si es el caso, nombre de la empresa operadora y los datos para contactar con la misma. La placa es responsabilidad exclusivamente del operador (adquirirla o elaborarla, grabar o hacer grabar la información requerida y fijarla a la aeronave) y en ella deberá constar «de forma legible a simple vista e indeleble» la información que se especifica. Por tanto, el tamaño de la placa ha de ser el necesario para que incluya toda la información requerida y ésta pueda leerse a simple vista.

Los drones de menos de $25 \mathrm{~kg}$ al despegue, no necesitan estar inscritos en el Registro de Matrícula de Aeronaves de AESA y disponer de certificado de Aeronavegabilidad.

Para poder realizar su trabajo aéreo sólo deberán cumplir las condiciones comunes para todos los drones:

Placa de identificación.

Piloto autorizado.

Operar en zonas no pobladas y en espacio aéreo no controlado.

Tendrán que volar dentro del alcanza visual del piloto y a una distancia de este no mayor de 500 metros y sin superar los 120 metros de altura.

No obstante, en el caso de que los drones sean inferiores a los 2 kilos al despegue, además podrán volar más allá del alcance visual del piloto, aunque tendrán que hacerlo dentro del alcance de la emisión por radio de la estación de control, y sólo podrán alcanzar una altura máxima de 400 pies $(120 \mathrm{~m})$. Pero para ello habrán de pedir a los Ser- 
vicios de Información Aeronáutica la emisión de un NOTAM, es decir un aviso al resto de los usuarios del espacio aéreo de dónde y cuándo va a volar, antes de realizar cualquier operación.

\section{CONCLUSIÓN: PROPUESTA PROPIA DE UN REGLAMENTO PARA ESPAÑA}

\section{Exposición de motivos}

Su Objetivo y necesidad de cobertura

La presente propuesta nace de una principal inquietud, cubrir una laguna jurídica, similar a la de otros precedentes que en España se conozcan al respecto, así el Proyecto del Real Decreto por el que se regula la utilización de las aeronaves pilotadas por control remoto que fue remitido por la Real Federación Aeronáutica española a la Dirección General de Aviación Civil el día 15 de julio del 2014 a los efectos del art. 24.1.c) de la Ley 50/1997, de 27 de noviembre.

La Disposición final segunda de habilitación normativa, en su segundo apartado, del Real Decreto Ley 8/2014, de 4 de julio, de aprobación de medidas urgentes para el crecimiento, la competitividad y la eficacia, puesta además en relación con el punto 4.15 de la Circular OACI 328 AN/190, pone de manifiesto que existe la necesidad de rellenar un vacío normativo que frena las inversiones económicas de un sector industrial, el aeronáutico, que sobre todo respecto a los pequeños aparatos está expectante del desarrollo de la norma que tenga en cuenta las peculiaridades por las que las autoridades integren finalmente ese tipo de aparatos aeronáuticos civiles en el espacio aéreo español no segregado y no sólo en el segregado como lo está siendo hasta el momento.

Desde este punto de vista podría decirse que el citado Real Decreto-Ley es una norma que ha nacido muerta si la finalidad que se pretende conseguir fuera esa integración que no existe hasta el momento, toda vez que para ello sea posible debería de ser modificada con otra norma con rango de ley. No obstante, aquí se pretende ya reglamentarla previendo ese futuro próximo, por lo que esta propuesta es diferente a la de la Real Federación Aeronáutica Española.

La aplicación civil en minis y micros sistemas de vuelos no tripulados constituye un «nicho de mercado» para pequeñas y medianas empresas, paradójicamente aquí más flexibles en costes que una gran 
industria. Piénsese que el solo hecho de no tener que pasar por la obligatoriedad de la certificación aeronáutica más exigente puede compensar ya su inversión. Para poder conseguirla se requiere antes resolver en el espacio aéreo ciertas dificultades. Así, el que sea una industria muy vinculada a consideraciones políticas y sólo generadora de altos costes y riesgos; la falta de integridad en la comunicación y control; la estandarización de procedimientos civiles y militares de aprendizaje; el establecimiento de corredores aéreos necesarios con la coordinación previa de vuelos; el de la interoperabilidad para el manejo de datos por la comunidad internacional; la gestión del espacio electromagnético; un nuevo concepto de aeronavegabilidad y de la reglamentación para la certificación; la determinación de requisitos médicos y de cualificación personal del operador, del mantenimiento de las plataformas, de los aspectos ligados al interfaz hombre-máquina y a la ergonomía de la estación; los contaminantes y el debido respeto a las reglas del aire y a las autoridades aeronáuticas en esa pretendida integración.

Por ello existe interés en el M. ${ }^{\circ}$ de Defensa de apoyar los desarrollos y necesidades industriales, pero de una forma centralizada, controlada y progresiva, es decir, que permita la transición desde un plan sólo ministerial a otro que sea un sistema de sistemas monitorizado por la Secretaría de Estado. En el caso de aeronaves civiles no tripuladas menores, así el de los drones tipo 1, la mayor limitación en el sistema es su miniaturización y autonomía de vuelo, de forma que las normas aplicables que aquí se postulan, de acuerdo con el considerando 5 del Reglamento CE núm. 216/2008 del Parlamento europeo y del Consejo, podrían tener cierta similitud a las reglas seguidas por los aficionados de radio-control, no registradas como leyes y sí como normas de sus clubes, basadas en consejos de otras organizaciones, entre ellas y sobre todo, la propia OACI y su comité conjunto de grupos de trabajo en cuanto que es la encargada de coordinar la elaboración de un documento de orientación estratégica que guíe la evolución normativa a nivel internacional y la elaboración de SARPs específicos en orden a la integración completa de los UAS en los aeródromos y el espacio aéreo en sus diversas clases.

Téngase en cuenta al respecto que junto con las aeronaves de Estado las aeronaves modelo -sólo dirigidas al uso recreativo-, según el punto 2.4 y 3.7 de la Circular OACI 328 AN/190, caen fuera de las disposiciones del Convenio de Chicago de 1944, por lo que son sólo reguladas por reglamentos nacionales. 
Como posibles aeronaves ya también de Estado que no por ello dejan de actuar en el mismo espacio, otros problemas relacionados que se manifiestan, por ejemplo, en el ámbito militar, están previstos por STANAGs (4586 y otros: 3377, 3809, 4250, 4545, 4559, 4575, $4607,4609,5500,7023,7024,7074,7085)$ y se relacionan con la falta de suficiente investigación en este sector, la ausencia de una economía de escalas y de una agencia central de adquisiciones que aumenta costes y ocasiona retrasos de entrega, la sobresaturación del espacio electromagnético de radio frecuencias, de frecuencias seguras para mando y control y ancho de banda, así como unas claras «Reglas de Enganche» o de «enfrentamiento» que deberán atender a maniobras de evasión, de rescate, de caza y ataque, etc. Por el contrario, las ventajas manifiestas son la reducción de riesgo con su empleo, su sencillez de transporte, despliegue, lanzamiento y recuperación, su gran autonomía y grado de control, la reunión de sensores y armamento en una sola plataforma, su flexibilidad y persistencia en las tareas, los ataques electrónicos, aire-superficie o aire-aire, así como la prevención común de colisiones y una infiltración mejorada en defensas enemigas por cuanto que permiten realizar misiones de gran distancia manteniendo a los operadores alertas y frescos hasta incrementar exponencialmente las capacidades militares conjuntas en las guerras «de cuarta generación», es decir, de entorno urbano y con todos los problemas que también ello conlleva si, entre otros, se atiende a su velocidad de tránsito o hipersónica, a los tendidos y centrales eléctricas, infraestructuras sensibles, el seguimiento de sospechosos terroristas o la pulcritud legalmente debida en la obtención y tratamiento de los datos obtenidos por grabación de cámaras aéreas.

Su nervio principal y peligro que se deriva del objetivo anterior:

Esta propuesta, que previamente requeriría de una ley, se concreta en pretender superar esa falta de integración de al menos los drones tipo 1 (de menos de 25 Kilogramos de peso) junto con el resto de aeronaves civiles de todo tipo en el espacio aéreo nacional. Lo hace, pues, para conseguir consolidar y potenciar el crecimiento industrial de un sector con posibilidades de todo tipo, militares y civiles o comerciales, siempre que se lleven a cabo operaciones con estos sistemas de un modo seguro.

Esta última idea es el nervio que ha servido de guía teniendo en cuenta además que en ocasiones una aeronave desarrollada en el ámbi- 
to de la industria de la defensa puede solicitar también en su caso el certificado de tipo civil a la autoridad civil competente y, en su caso, a otras extranjeras, por lo que la normativa técnica de ambos campos deberá tener una base común. También lo será para la aplicación e interpretación futura de la norma si se diera el caso. Hoy por hoy no resulta exagerado comprender que incluso el uso de tales drones en los conflictos armados, o por la simple delincuencia, puede ser como el de un videojuego con víctimas reales, peligro que en todo caso se trata de evitar.

La normativa internacional de referencia y la contribución española

Para ello se es consciente de que el marco normativo internacional de referencia lo proporciona la Organización de Aviación Civil (OACI) como agente que coordina y armoniza términos, estrategias y principios, para garantizar la seguridad y uniformidad de la aviación civil internacional.

Hasta el momento esa norma de referencia la constituye la Circular 328 AN/190, de cuya doctrina, definiciones y glosario de expresiones esta propuesta se hace eco de conformidad con lo dispuesto en la letra c) de su párrafo 1.6, esto es, la de proporcionarle estatalmente a la OACI «información sobre sus propias experiencias relacionadas con estas aeronaves", toda vez que si bien se parte de la base de que este tipo de artefactos forman parte del sistema de transporte aéreo, local o global, y quedan sometidos a las reglas aeronáuticas generales, por su carácter transversal en determinados aspectos requieren una propia regulación por respeto a otras disciplinas afectadas, así el caso de la privacidad de datos, materia donde debe de ser asumida por analogía toda su regulación conformada por el Convenio para la protección de las personas respecto al tratamiento de datos de carácter personal de 28.01.1981(arts. 5,6,8 y 9), en la Directiva sobre protección de datos de 1995 (Considerandos $13 .{ }^{\circ}, 16 .^{\circ}, 43 .^{\circ}$ y art. 13), en el Reglamento (CE) 45/2001 (considerando $18 .^{\circ} \mathrm{y}$ art. 20) del Parlamento Europeo y del Consejo de 18.12.2000, así como ya en España a través de la Ley Orgánica 15/1999, de 15 de diciembre, de Protección de Datos (art. 2) y en la Ley orgánica 4/1997, de 4 de agosto, por la que se regula la utilización de videocámaras por las fuerzas y cuerpos de seguridad en lugares públicos. 
La definición española de "vehículo aéreo no tripulado» y sus requisitos

Tiene su antecedente en la que viene definida sólo para el ámbito restringido del tránsito aéreo militar, concretamente en la Orden PRE/1366/2010, de 20 de mayo, por la que se modifica el Reglamento de Circulación Aérea Operativa (BOE núm. 130 de 28.05.10), pero completándola con la importante apreciación de que son entes complejos que, como entidad única, no sólo están integrados por la propia aeronave sino por la estación remota, los enlaces de comunicación y el equipo de lanzamiento y recogida como mínimo.

Teniendo en cuenta, además, el fin de esta propuesta así como minimizar los conflictos con las aeronaves convencionales y de que tengan la misma seguridad que éstas, a los requisitos tradicionales de ser capaz de mantenerse aerodinámicamente en vuelo, ser pilotado en forma remota o incluyendo un programa de vuelo automático, ser reutilizable y no ser un arma guiada o similar para lanzamiento de otras armas, deberán de reunir en su caso las certificaciones de las características de la aeronave, y en todo momento las licencias de mantenimiento de personal y las licencias del personal que las opera en cuanto que, siendo los titulares de la licencia de su explotación, se hacen responsables de la aeronave y de los daños que cause.

A tal efecto, igual que las convencionales, deben reunir en un principio los mismos requisitos de publicidad relativos a la bandera de su matrícula, el nombre, el titular y otros datos en cuanto a su forma, caracteres, colocación y emplazamiento, debiendo tenerse en cuenta a estos efectos la Orden de 22.09.1977 sobre Reglamento de Marcas de nacionalidad y de matrícula de las aeronaves civiles y la Resolución de la Subsecretaría de Aviación Civil Internacional de 17.11.1977 que aprueba el Texto Refundido del Reglamento de marcas de nacionalidad y matrícula de aeronaves civiles (BOE núm. 7 de 09.01.1978).

No obstante, si bien de acuerdo con los párrafos 6.24 y 6.25 de la Circular 328 OACI las marcas de la aeronave deberán ser identificables en casos de estrecha proximidad con otras aeronaves, en caso de interceptación, o en caso de aterrizaje en aeródromo distinto al designado, se prevé expresamente para el caso de aeronaves no tripuladas pequeñas -caso del dron tipo 1- el que "pueda ser necesario» establecer la exención para las marcas o sistemas alternativos, como el etiquetado que ya se utiliza para las partes de aeronave y que permite una identificación adecuada. 
Su matriculación

Para poder matricularlas en España se prevé un régimen de doble inscripción registral materializado a través del Registro de Matrícula de Aeronaves y el Registro de Bienes Muebles, afectándole internacionalmente a España para su inscripción el Convenio relativo a garantías internacionales sobre elementos de equipo móvil que fue firmado en Ciudad del Cabo (Sudáfrica) el 16.11.2001. No obstante, conforme al art. 151 de la Ley 48/1960, de 21 de julio, de Navegación Aérea, las aeronaves pilotadas por control remoto de hasta $25 \mathrm{kgs}$ de masa máxima al despeque están exceptuadas de los requisitos de inscripción en el Registro de Matrícula de Aeronaves civiles y de la obtención del certificado de aeronavegabilidad a que se refieren los arts. 29 y 36 de aquella ley, estando sujetas a condiciones específicas para su pilotaje.

Su circulación aérea

Como requisito externo para su operatividad en espacios no segregados debe de estar conforme a las reglas del Aire (Anexo 2 del Convenio de Chicago de 1944) que están basadas en el principio de responsabilidad del piloto de «ver y evitar» (see and avoid), ya sea volando visualmente (VFR) o por instrumentos (IFR).

Por ello tales aviones tienen que estar provistos de los servicios de control de tránsito aéreo, es decir, el de su asesoramiento e información de vuelo (incluyendo las condiciones meteorológicas), debiéndose el piloto atenerse con precisión al plan de vuelo previamente comunicado, al derecho de preferencia de paso en un espacio tridimensional como el aéreo, a la ruta y altitud, e informar por último de su posición al controlador en todo momento.

Tales postulados, de acuerdo con los párrafos 2.2,3.1 y 3.3 de la Circular 328 OACI deberán a su vez ser complementados con las normas y métodos específicamente recomendados (SARPs) y publicados en el conjunto de Anexos Técnicos al Convenio de Chicago no tanto para aeronaves no tripuladas (UAS) sino sólo ya como «aeronaves pilotadas a distancia» (RPA), distinción que se hace para resaltar que en todo caso debe de haber un piloto responsable de la operación del sistema de vuelo no tripulado y que en ninguna circunstancia puede ser sustituido por tecnología alguna y futura previsible. 
Sobrevuelo de otros Estados y régimen aeroportuario

En aplicación de lo dispuesto en el art. 8 del Convenio de Chicago se prevé expresamente para este tipo de aeronaves, igual que para las tripuladas, que «ninguna aeronave capaz de volar sin piloto volará sobre el territorio de un Estado contratante, a menos que se cuente con autorización especial de tal Estado y de conformidad con los términos de dicha autorización. Cada Estado contratante se compromete a asegurar que los vuelos de tales aeronaves sin piloto en las regiones abiertas a la navegación de las aeronaves civiles sean controlados de forma que se evite todo peligro a las aeronaves civiles». Por tanto, les resultan de la misma forma aplicables las libertades del aire y los Convenios bilaterales o multilaterales que hayan suscrito los Estados.

Esto implica que el piloto de estos drones deberá ser capaz de cumplir las instrucciones proporcionadas por el Estado que sobrevuele, incluso por medios electrónicos o visuales, y tener la capacidad de dirigirse al aeropuerto (o aeródromo) especificado a petición del Estado que se lo requiera en virtud del ejercicio de su soberanía salvaguardada por los arts. 3 bis del Convenio de Chicago y 4.2 de la Circular 328 OACI. Aunque hasta el momento en esos Estados las operaciones de estos drones en sus aeropuertos aún no fueran viables y siguiesen llevándose a cabo de forma segregada, de acuerdo con el punto 4.10 de la Circular 328 OACI sus Estados contratantes tienen la libertad de permitir operaciones civiles de UAS sólo, desde y hacia aeródromos designados, siempre que no se introduzca discriminación con respecto a la matrícula nacional o extranjera de las aeronaves.

La Responsabilidad por daños e investigación de accidentes graves

En este ámbito, si la aeronave es extranjera, rige el Convenio de Roma de 07.10.1952 (CR 1952) sobre daños causados a terceros en la superficie por aeronaves extranjeras, que establece un régimen de responsabilidad cuasi-objetiva.

En cambio, si fuera nacional, la propia Ley de Navegación Aérea en sus arts. 111 y siguientes la contempla estableciendo ya un régimen más severo de responsabilidad objetiva.

De acuerdo con esa normativa legal es imperativo el establecimiento de un seguro (art. 127 de la LNA) y, por tanto, cualquier RPA deberá contar preceptivamente de un seguro con daños a terceros si se quiere volar con él. No obstante, por los motivos que se dirán no es deseo de esta propuesta acoger este sistema de responsabilidad por 
daños, motivo por el cual lo entiende transitoriamente vigente hasta que haya una reforma legal al respecto. Se significa que ese desarrollo normativo no es propio de esta propuesta puesto que tiene que tener un carácter legal y no meramente reglamentario, más si cabe cuando es la Ley de Seguridad Aérea la que de acuerdo con el principio de legalidad sancionador debe de castigar con ese rango jerárquico normativo la explotación de un UAS sin contar con un seguro obligatorio.

Esa reforma, entre otros posibles aspectos, debiera corregir el único establecimiento actual de una correlación directa entre la masa de la aeronave y el importe máximo de la indemnización. La reforma legal que desde esta misma propuesta reglamentaria se sugiere deberá de ponderar ese sistema sabiendo que (1) las consecuencias de un impacto no dependen únicamente del peso de la aeronave sino de la energía cinética, esto es, de la masa y la velocidad, (2) que el uso de UAS en el espacio aéreo no segregado incrementa las probabilidades de que se produzcan no tanto grandes impactos contra el terreno, que también, sino sobre todo colisiones en el aire, más si cabe cuanto mayor sea la altitud, así como que (3) de acuerdo con la doctrina científca autorizada el tratamiento de tal clase de impactos merecería una clasificación y diferenciarse los daños a efectos indemnizatorios según las diferentes variables de sus causas al respecto (así si se colisiona en terreno despoblado o no y en función de la distinta densidad de población), es decir, atendiendo no sólo al peso o masa de la aeronave sino también a su volumen y velocidad de marcha.

Asimismo, de acuerdo con la experiencia como la que sucedió tras los atentados del 11-S tampoco es recomendable someter sin más la responsabilidad del operador del UAS a la responsabilidad extracontractual genérica e ilimitada del art. 1902 y siguientes del Código Civil porque desincentivaría al operador no sólo la asunción de un riesgo que es propio a su trabajo, encarecería las primas del seguro y, por ende, la paralización de flotas por no ser viable su cobertura aseguradora.

Ese seguro obligatorio aún requiere de un desarrollo normativo al estilo del Reglamento (CE) núm. 785/2004 pero aún más perfeccionado. Deberá precisar el concepto de «siniestro» que da lugar a su cobertura temporal y saber así las reclamaciones que debe de hacer frente la compañía de seguros.

En cuanto a la investigación técnica de accidentes e incidentes graves se hace una remisión al Reglamento (UE) núm. 996/2010, de 20 de octubre, del Parlamento Europeo y del Consejo, al Ane- 
xo 13 del Convenio de Chicago y la Circular 328 OACI, donde el párrafo 5.41 establece a modo de mejoras técnicas y de lecciones aprendidas del sector el deber de compartir en la medida de lo posible con los demás Estados los datos que por ellas se recojan, de forma que sus informes están disponibles en la página web del NTSB (National Transport Safety Board).

Por tanto, en su virtud,

Teniendo en cuenta así la normativa que está previamente recogida para el desarrollo legal futuro en el espacio aéreo no segregado en España de "drones" civiles de pequeño peso (Tipo 1) con funciones de vigilancia aérea y de colaboración de lucha antiterrorista por motivos de seguridad en infraestructuras sensibles, de acuerdo con la habilitación normativa prevista en la Disposición final segunda de habilitación normativa, en su segundo apartado, del Real Decreto Ley 8/2014, de 4 de julio, de aprobación de medidas urgentes para el crecimiento, la competitividad y la eficacia, y sin perjuicio de que pronto haya una nueva ley que permita su integración en el espacio aéreo no segregado, a propuesta de la Ministra de Fomento, con la aprobación previa del Ministerio de Hacienda y Administraciones Públicas, (...) con el Consejo de Estado y previa deliberación del Consejo de Ministros en su reunión del día (...), la presente propuesta de ley y, ya más concretada, de su reglamento, se concreta en el siguiente articulado que

\section{DISPONGO}

\section{TÍTULO PRELIMINAR}

\section{Disposiciones Generales}

\section{Art. 1. Objeto}

Tiene por objeto establecer las condiciones actuales de uso y operación de los sistemas de aeronaves pilotadas por control remoto menores a $25 \mathrm{~kg}$ (drones tipo 1) en el espacio aéreo no segregado y como desarrollo de la medida V del Real Decreto Ley 8/2014, de 4 de julio, de aprobación de medidas urgentes para el crecimiento, la competitividad y la eficacia, pero con las perspectivas futuras de que no sólo lo sean para el espacio aéreo segregado sino también para el espacio aéreo no segregado cuando la reforma de la actual ley así lo permita, siendo el presente texto un adelanto provisional de la misma 
en cuanto que, una vez promulgada, también exigirá su consiguiente desarrollo, adaptación y reforma con respecto a aquélla.

\section{Art. 2. Principios que inspiran este Reglamento}

2.1 Se parte de dos postulados formulados en el Reglamento del Parlamento europeo núm. 216/2008 en sus consideraciones 5 y 29, según los cuales no resulta adecuado someter a todas las aeronaves a normas comunes pero sí proporcionadas al nivel de seguridad requerida tanto para la aviación civil en general como a la protección del medio ambiente, de forma que para alcanzar tales objetivos tienen un papel preponderante los Estados miembros de la UE y ésta otro ya que lo es sólo subsidiario.

2.2 La prioridad es garantizar la seguridad operacional de todos los usuarios del espacio aéreo así como la seguridad de las personas y bienes en tierra.

\section{Art. 3. La definición española de "vehículo aéreo no tripulado» y sus requisitos}

3.1 A los efectos de esta propuesta es el »vehículo aéreo propulsado que no lleva personal como operador a bordo y que, como ente complejo y único, no sólo está integrado por la propia aeronave» sino, como mínimo, por la estación remota, los enlaces de comunicación y el equipo de lanzamiento y recogida.

3.2 Para poder operar actualmente sólo en espacio aéreo segregado y minimizar así los conflictos con las aeronaves convencionales debe reunir como requisitos los siguientes: (1) el de ser capaz de mantenerse aerodinámicamente en vuelo, (2) ser pilotado en forma remota o incluyendo un programa de vuelo automático, (3) ser reutilizable y (4) no ser un arma guiada o similar para lanzamiento de otras armas.

\section{Art. 4. Alcance y Actividad que regula}

Esta propuesta de reglamento regula en España los vuelos no tripulados (UAV o RPAS) para drones tipo 1 con misiones como las de vigilancia y de seguridad en lugares públicos o privados que por su carácter estratégico, de infraestructura sensible u otro interés general similar haga necesario el establecimiento público o privado de dicho servicio.

Se aplica a los sistemas de aeronaves pilotadas por control remoto, a su aeronavegabilidad, a las operaciones que se realicen con ellos $\mathrm{y}$ al personal que las pilote o auxilie poder hacerlo. 
No se aplica a drones que no sean tipo 1 ni tengan aquellas misiones, o éstas sean militares, a globos científicos y a los cautivos y a los vuelos íntegramente realizados en espacios interiores entendidos como recintos cerrados.

\section{TÍTULO I}

\section{CAPÍtULO I}

\section{Operatividad locativa actual y la futura prevista}

\section{Art. 5. Lugares donde actualmente puede tener lugar su vuelo}

\subsection{Zonas no pobladas}

5.1.1 Cuando tal tarea sea privada y conlleve la realización de trabajos aéreos como los citados, incluyendo filmación y grabación, las operaciones nocturnas y diurnas que conforme a la normativa de protección de datos, aeronáutica y medioambiental se puedan realizar, se limitarán a las zonas no pobladas limítrofes donde exista una señal (visual, electromagnética o acústica) que a tales efectos las delimite, dentro del alcance visual del piloto, a una distancia de éste no mayor de $500 \mathrm{~m}$. y a una altura sobre el terreno no mayor de 400 pies (es decir, como máximo $120 \mathrm{~m}$. sobre el terreno).

\subsubsection{En caso contrario, necesitarán la correspondiente auto-} rización que, sin embargo, no necesitarán las Fuerzas y Cuerpos de seguridad para llevar a cabo esa misma tarea en su versión ya pública. Esa autorización se presumirá otorgada cuando en circunstancias especiales de seguridad socialmente acreditadas tales como siniestros, catástrofes, delitos o atentados, colaboren o sean requeridas por aquéllas para una determinada operación de interés general.

5.1.3 En caso de esa colaboración sólo deberán de comunicarlo a la autoridad competente si no han sido antes requeridas.

\subsection{Zonas pobladas}

El uso privado de tales drones con el mismo fin anterior en zonas ya pobladas tales como ciudades o sobre aglomeraciones, manifestaciones, fiestas o conciertos tiene como única excepción el que tenga lugar en recintos completamente cerrados (incluyendo el techo) y 
siempre que no haya impedimentos medioambientales de acuerdo con la tramitación de la normativa autonómica y local prevista al efecto ${ }^{2}$.

\section{CAPÍTULO II}

\section{Operatividad de pilotaje}

\section{Art. 6. Requisitos personales de pilotaje: Registro de AESA (comunicación y autorización)}

6.1 La habilitación como operador de drones civiles para realizar este tipo de trabajos requiere de una comunicación previa (que no de autorización) para tiempo indefinido y una declaración responsable, por lo que es necesario tan sólo un acuse de recibo una vez que se presente en el Registro de AESA junto con la documentación exigida, cuyo acuse de recibo le habilitará como operador de drones civiles.

La comunicación previa y su modificación en su caso deberán contener ${ }^{3}$ :

a) Los datos identificativos del operador, de la aeronave que vaya a utilizarse, del piloto/s que realice la operación y sus condiciones acreditativas.

${ }^{2}$ Cuando la nueva ley (que sugerimos) permita su operatividad en el espacio aéreo no segregado, se debieran de tener en cuenta la configuración de corredores aéreos seguros en función de la altitud marginal mínima y máxima donde se pretendan que circulen, las líneas eléctricas, obstáculos existentes, infraestructuras terrestres, la cartografía urbana y rural, así como el espacio de seguridad de tres dimensiones que deban de tener con respecto otras aeronaves y artefactos aéreos.

Para esta clase de drones tipo 1, salvo mejor estudio, la altitud referida de tales pasillos aéreos podría ser de un mínimo de 50 a 120 metros de altitud continuada con respecto a la orografía de la superficie que sobrevuelen, debiendo de preverse algún espacio de seguridad alternativo sólo para su excepcional uso comunicado en el tráfico ordinario de los drones, tráfico que en todo caso deberá estar limitado en su densidad espacial con arreglo también a otros factores como los contaminantes y sobre todo la finalidad de su misión y servicio, dado que por tratarse el espacio aéreo no segregado de un dominio público tendrán preferencia los que sean de Estado y con funciones de aviso, interceptación, y en su caso, hasta derribo en lugar despoblado, de acuerdo con las reglas de enganche que se formalicen al respecto por cuestiones lógicas de seguridad e interés general.

${ }^{3}$ En el futuro, cuando la ley que se sugiere realizar prevea su vuelo en espacio aéreo no segregado será recomendable que estipule que se haga un estudio aeronáutico previo de la frecuencia de vuelos que a tenor de sus misiones tengan prevista fuera ya de su sector aéreo en principio pretendido, controlado y asignado, así como de sus horarios y lugares de despegue y de aterrizaje, en cuyo caso no bastará la simple comunicación con el fin de poder regular adecuadamente la densidad de tráfico aéreo del parque autorizado de toda la flota de drones, es decir, con los vuelos concretos ya previstos y concedidos. 
b) Las características de la aeronave, su configuración y prestaciones.

c) El tipo de operación.

d) Sus condiciones de seguridad y limitaciones si las tiene.

6.2 En todo caso para la realización de fotografías o filmaciones con cualquier tipo de aeronaves, tripuladas o no, es necesario obtener una autorización específica de AESA (Orden de Presidencia del Gobierno de 14 de marzo de 1957), en cuya página web figura la información e instrucciones al respecto, en concreto el Procedimiento para la obtención de permisos de vuelo de trabajos aéreos.

6.3 Todos los pilotos de drones, indistintamente del tamaño de la aeronave, deberán acreditar una serie de requisitos:

A) En primer lugar, acreditar que posee los conocimientos teóricos necesarios para obtener una licencia de piloto. Se puede hacer de tres formas:

- tener o haber tenido (en los últimos 5 años) una licencia de piloto (cualquier licencia), pudiendo incluirse la de planeador, de globo o de ultraligero;

- demostrar de forma fehaciente que disponen de los conocimientos teóricos para obtenerla (por medio certificado de conocimientos teóricos emitido por una organización de formación aprobada por AESA, ATO, o, en el caso de que esos conocimientos correspondan a una licencia de piloto de ultraligero, mediante un certificado individual como APTO tras realizar el correspondiente examen oficial de conocimientos teóricos),

— o si el peso máximo al despegue no es superior a $25 \mathrm{~kg}$ por medio de un certificado básico o avanzado emitido por una organización de formación aprobada (ATO) tras superar un curso al efecto. Esta última podría ser en el caso del Proyecto.

La lista de organizaciones de formación aprobadas puede encontrarse en la página web de AESA.

Además, si no tuvieran una licencia de piloto, deben acreditar que tienen más de 18 años.

B) En segundo lugar, por su peso deberán presentar un determinado certificado médico, el de Clase LAPL (para aeronaves de hasta $25 \mathrm{~kg})$. 
C) Finalmente deberán acreditar que disponen de los conocimientos adecuados de la aeronave que van a pilotar y de su pilotaje, por medio de un documento que puede ser emitido por el operador, por el fabricante de la aeronave o una organización autorizada por éste, o por una organización de formación aprobada.

\section{CAPÍTULO III}

\section{Operatividad del explotador $u$ operador.}

\section{Art. 7. Requisitos de la empresa operadora propietaria y responsable de los drones}

Indistintamente del tamaño de la aeronave tripulada por control remoto, todos los operadores de drones deberán cumplir los siguientes requisitos:

a) Disponer de la documentación sobre la caracterización de la aeronave (configuración, prestaciones).

b) Contar con un Manual de operaciones en el que establezca los procedimientos de la operación (por ejemplo, criterios para designar las zonas de despegue y aterrizaje, de condiciones meteorológicas para poder volar, etc.).

c) Haber realizado un estudio aeronáutico de seguridad de la operación.

d) Establecer un programa de mantenimiento de la aeronave.

e) Tener un piloto que cumple los requisitos establecidos.

f) Disponer de un seguro conforme a la normativa vigente.

g) Adoptar las medidas adecuadas para que la aeronave no sufra actos de interferencia ilícita durante las operaciones, incluyendo interferencias deliberadas al enlace de radio y acceso no autorizado a la estación de control, durante el vuelo, o a la ubicación donde se almacene la aeronave.

h) Garantizar que la operación se realice a una distancia mínima de 8 kilómetros de cualquier aeropuerto o aeródromo, o de 15 si en él se puede operar en vuelo instrumental, o caso contrario haberse puesto de acuerdo con el mismo. Sin embargo, según ya vimos, en el caso de operadores de aeronaves de hasta 25 kilos de peso máximo al despegue -como es el nuestro- no necesitan solicitar una autorización para poder operar, únicamente tienen que presentar en AESA una comunicación previa y declaración responsable conforme su aeronave 
cumple todas esas exigencias, junto con la documentación que lo acredite:

- Esa comunicación previa y declaración responsable habrán de presentarse a AESA con una antelación mínima de 5 días antes del inicio previsto de la operación.

- AESA facilitará al interesado un acuse de recibo que servirá para acreditar ante terceros que el interesado está legalmente habilitado para realizar esa actividad.

- Cualquier modificación que se produzca también deberá ser notificada a AESA con una antelación mínima de 5 días, presentando la documentación original que haya sido modificada junto con declaración responsable que cubra las modificaciones introducidas.

\section{TÍTULO II}

\section{CAPÍtULO ÚNICO}

\section{Aeronavegabilidad}

\section{Art. 8. Requisitos de vuelo}

8.1 Todos los drones, sin excepción, deben de llevar fijada en su estructura una placa de identificación en la que deberá constar, de forma legible y a simple vista e indeleble, la identificación de la aeronave, mediante la designación específica, número de serie si es el caso, nombre de la empresa operadora y los datos para contactar con la misma. La placa es responsabilidad exclusivamente del operador (adquirirla o elaborarla, grabar o hacer grabar la información requerida y fijarla a la aeronave) y en ella deberá constar «de forma legible a simple vista e indeleble» la información que se especifica. Por tanto, el tamaño de la placa ha de ser el necesario para que incluya toda la información requerida y ésta pueda leerse a simple vista.

La estación de control en tierra llevará fijada, a su vez, una placa con la misma información.

8.2 Los drones de menos de $25 \mathrm{~kg}$ al despegue, tipo 1 , como es aquí el caso objeto de propuesta de regulación reglamentaria, no necesitan estar inscritos en el Registro de Matrícula de Aeronaves de AESA y disponer de certificado de Aeronavegabilidad previsto en el art. 29 y 36 de la Ley 48/1960, de 21 de julio, de Navegación Aérea. 
8.3 Hasta que no entre en vigor la ley que permite su vuelo en espacio aéreo no segregado, para poder realizar su trabajo aéreo sólo deberán cumplir las condiciones comunes para todos los drones:

Placa de identificación.

Piloto autorizado.

Operar en zonas no pobladas y en espacio aéreo no controlado.

Tendrán que volar dentro del alcanza visual del piloto y a una distancia de este no mayor de 500 metros y sin superar los 120 metros de altura.

No obstante, en el caso de que los drones sean inferiores a los 2 kilos al despegue, además podrán volar más allá del alcance visual del piloto, aunque tendrán que hacerlo dentro del alcance de la emisión por radio de la estación de control, y sólo podrán alcanzar una altura máxima de 400 pies $(120 \mathrm{~m})$. Pero para ello habrán de pedir a los Servicios de Información Aeronáutica la emisión de un NOTAM, es decir un aviso al resto de los usuarios del espacio aéreo de dónde y cuándo va a volar, antes de realizar cualquier operación.

\section{Art. 9. Vuelos internacionales}

9.1 Igual que para las tripuladas volará sobre el territorio de un Estado contratante con autorización de tal Estado y de conformidad con los términos de dicha autorización.

9.2 En las regiones abiertas a la navegación de las aeronaves civiles serán controladas de forma que se evite todo peligro a las aeronaves civiles.

9.3 Les resultan aplicables las libertades del aire y los Convenios bilaterales o multilaterales que hayan suscrito los Estados.

9.4 El piloto deberá ser capaz de cumplir las instrucciones proporcionadas por el Estado que sobrevuele, incluso por medios electrónicos o visuales, y tener la capacidad de dirigirse al aeropuerto especificado a petición del Estado que se lo requiera en virtud del ejercicio de su soberanía salvaguardada por los arts. 3 bis del Convenio de Chicago y 4.2 de la Circular 328 OACI.

9.5 De acuerdo con el punto 4.10 de la Circular 328 OACI, y hasta su plena integración con el espacio aéreo no segregado, los Estados contratantes tienen la libertad de permitir operaciones civiles de UAS sólo, desde y hacia aeródromos designados, siempre que no 
se introduzca discriminación con respecto a la matrícula nacional o extranjera de las aeronaves.

\section{TÍTULO III}

\section{ACCIDENTES E INCIDENTES AÉREOS}

\section{Art. 10. Responsabilidad por daños}

$10.1 \mathrm{El}$ operador adoptará las medidas necesarias para garantizar la seguridad de la operación y para la protección de las personas y bienes no sólo subyacentes.

10.2 En cuanto a la investigación técnica de accidentes e incidentes graves existe el deber de compartir en la medida de lo posible con los demás Estados los datos que por ellas se recojan.

10.3 En cuanto al servicio de meteorológico y de seguridad de la aviación se atenderá a los puntos 5.27-5.36 de la Circular 328. Habrá de preverse la estela de turbulencias y no sólo las que sean naturales, así como los posibles sabotajes e interferencias ilícitas mal intencionadas y las zonas de acceso restringido.

\section{Art. 11. Seguros y derecho de paso}

11.1 Cualquier RPA deberá contar preceptivamente de un seguro con daños a terceros.

11.2 En cuanto al derecho de paso entre aeronaves será similar al tráfico de superficie teniendo en cuenta su movimiento tridimensional, de forma que:

- cuando dos aeronaves converjan a un nivel aproximadamente igual, la que vuela a la derecha tiene derecho de paso, salvo que los aviones deban ceder el paso a los dirigibles, planeadores y globos, así como a las aeronaves que remolquen objetos.

- cuando una aeronave alcance a otra, debe cederle el paso variando el rumbo hacia la derecha.

- cuando dos aeronaves se acerquen de frente, ambas deben variar el rumbo hacia la derecha.

\section{Disposición Transitoria Única. Normas transitorias}

La presente propuesta es, de hecho, una disposición transitoria hasta que no se regule por ley el vuelo de drones en espacios aéreos 
no segregados, y tiene apuntadas en algunas de sus disposiciones las previsiones de futuro al respecto para su estudio.

Asimismo se prevé un plazo prudencial de tres meses para la adaptación de los operadores a esta norma una vez que en su caso entrara en vigor.

\section{Disposición Final Primera. Medidas de ejecución}

1. Por Resolución del Director de la Agencia Estatal de Seguridad Aérea, publicada en el BOE, se podrán establecer los medios aceptables de cumplimiento para la acreditación de los requisitos aquí previstos y de sus disposiciones de desarrollo.

2. La Agencia Estatal de Seguridad Aérea podrá publicar en su página web cualquier documentación orientativa para la aplicación de esta propuesta, una vez formalizada en la norma que con el rango corresponda, y de sus disposiciones de desarrollo.

\section{Disposición Final Segunda. Habilitación normativa}

Por orden del Ministerio de Fomento se podrán dictar las disposiciones de desarrollo de esta norma.

\section{Disposición Final Tercera. Título competencial}

Esta norma se dicta al amparo de la competencia exclusiva que atribuye al estado el art. 149.1.20. ${ }^{\mathrm{a}}$ de la CE en materia de control del espacio aéreo, tránsito y transporte aéreo.

\section{Disposición Final Cuarta}

La presente propuesta se propondrá a M. ${ }^{\circ}$ de Fomento a través de los posibles clientes del Proyecto para tramitar su Expediente de aprobación y orientarse en la fecha que se pueda prever de entrada en vigor de la norma si fuera el caso.

\section{Disposición Adicional Única}

En ese expediente se deberán abordar los Informes relativos a la transversalidad de otras materias afectadas y que, sobre todo, estén reguladas por Ley (infracciones y sanciones, datos, etc.). 
UN INICIO DE LA ARMONIZACIÓN PRETENDIDA: EL GLOSARIO DE DEFINICIONES RECONOCIDAS POR LA OACI

Aeronave*. Toda máquina que pueda sustentarse en la atmósfera por reacciones del aire que no sean las reacciones del mismo contra la superficie de la tierra.

Aeronave (categoría de)*. Clasificación de las aeronaves de acuerdo con características básicas especificadas, por ejemplo: avión, helicóptero, planeador, globo libre.

Control operacional*. Autoridad ejercida respecto a la iniciación, continuación, desviación o terminación de un vuelo en interés de la seguridad de la aeronave y de la regularidad y eficacia del vuelo.

Explotador*. Persona, organización o empresa que se dedica, o propone dedicarse, a la explotación de aeronaves.

Miembro de la tripulación*. Persona a quien el explotador asigna obligaciones que ha de cumplir a bordo, durante el período de servicio de vuelo.

Miembro de la tripulación de vuelo*. Miembro de la tripulación, titular de la correspondiente licencia, a quien se asignan obligaciones esenciales para la operación de una aeronave durante el período de servicio de vuelo.

Pilotar*. Manipular los mandos de una aeronave durante el tiempo de vuelo.

Piloto al mando*. Piloto designado por el explotador, o por el propietario en el caso de la aviación general, para estar al mando y encargarse de la realización segura de un vuelo.

Tiempo de vuelo-aviones*. Tiempo total transcurrido desde que el avión comienza a moverse con el propósito de despegar, hasta que se detiene completamente al finalizar el vuelo.

Tiempo de vuelo - helicópteros *. Tiempo total transcurrido desde que las palas del rotor comienzan a girar hasta que el helicóptero se detiene completamente al finalizar el vuelo y se paran las palas del rotor.

Tiempo de vuelo por instrumentos*. Tiempo durante el cual se pilota una aeronave solamente por medio de instrumentos sin referencia a puntos externos. 


\section{LAS ABREVIATURAS Y ACRÓNIMOS DE OACI}

ACAS Sistema anticolisión de a bordo

ADS-B Vigilancia dependiente automática - radiodifusión

AM(R)S Servicio móvil aeronáutico (r)

ARNS Servicio de radionavegación aeronáutica

ARNSS Servicio de radionavegación aeronáutica por satélite

ATC Control del tránsito aéreo

ATM Gestión de tránsito aéreo

ATS Servicios de tránsito aéreo

CAA Autoridad de Aviación Civil

C2 Mando y control

C3 Mando, control y comunicaciones

CMR Conferencia Mundial de Radiocomunicaciones

CPDLC Comunicaciones por enlace de datos controlador-piloto

EASA Agencia Europea de Seguridad Aérea

EUROCAE Organización europea para el equipamiento de la aviación civil

HF Alta frecuencia

IFR Reglas de vuelo por instrumentos

OACI Organización de Aviación Civil Internacional

PANS Procedimientos para los servicios de navegación aérea

QOS Calidad de servicio

RPA Aeronave pilotada a distancia

RPAS Sistema de aeronave pilotada a distancia

RTCA, Inc.

SAR Búsqueda y salvamento

SARPS Normas y métodos recomendados

SATCOM Comunicación por satélite

SMAS(R) Servicio móvil aeronáutico $(\mathrm{R})$ por satélite 
SMS Sistema de gestión de la seguridad operacional

SSP Programa estatal de seguridad operacional

UA Aeronave no tripulada

UAS Sistema(s) de aeronave(s) no tripulada(s)

UAV Vehículo aéreo no tripulado (término obsoleto)

UIT Unión Internacional de Telecomunicaciones

UOC Certificado de explotador UAS

VDL Enlace digital en VHF

VFR Reglas de vuelo visual

VHF Muy alta frecuencia

VLOS Visibilidad directa visual

VMC Condiciones meteorológicas de vuelo visual

\section{FIGURAS DE ANEXO SOBRE PROBLEMÁTICAS OPERATIVAS}

\section{Figura 1. Visibilidad directa visual}

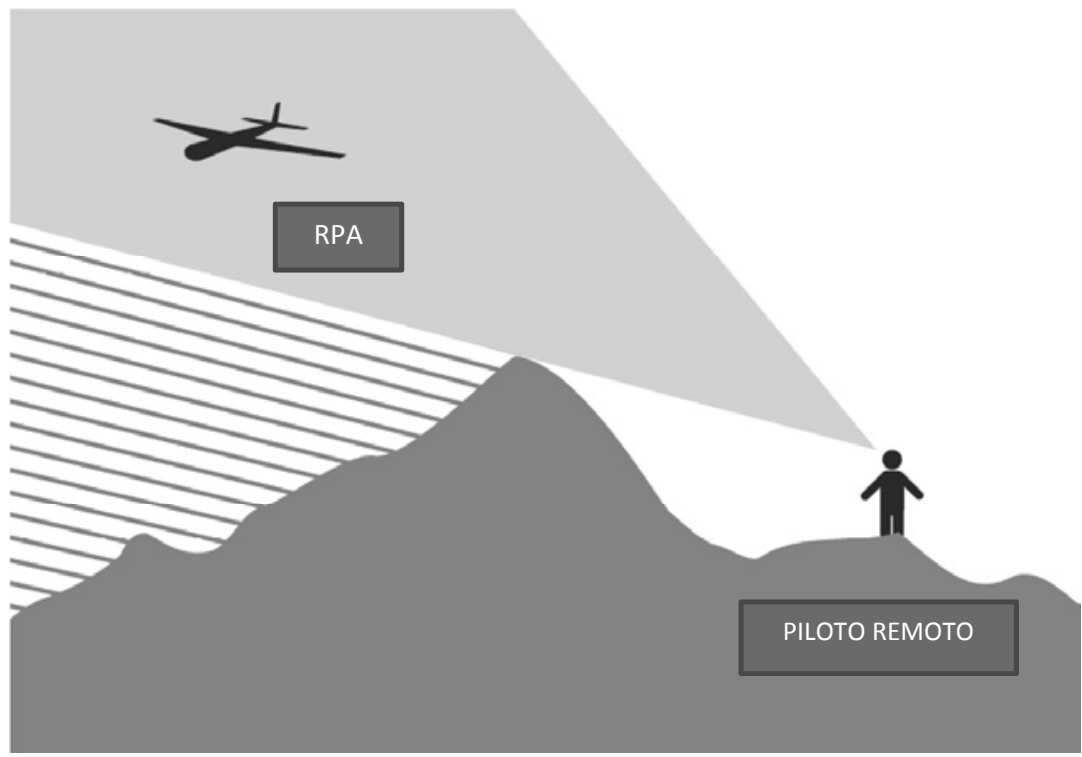




\section{Figura 2. Detectar y evitar}

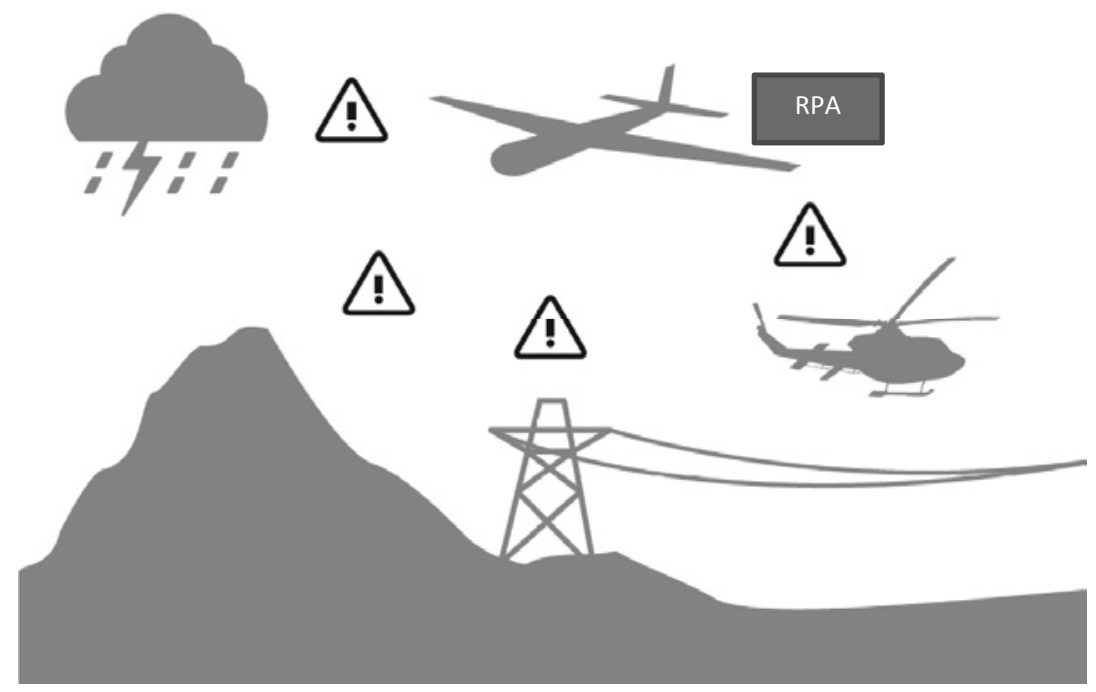

Figura 3. Enlace de comunicaciones

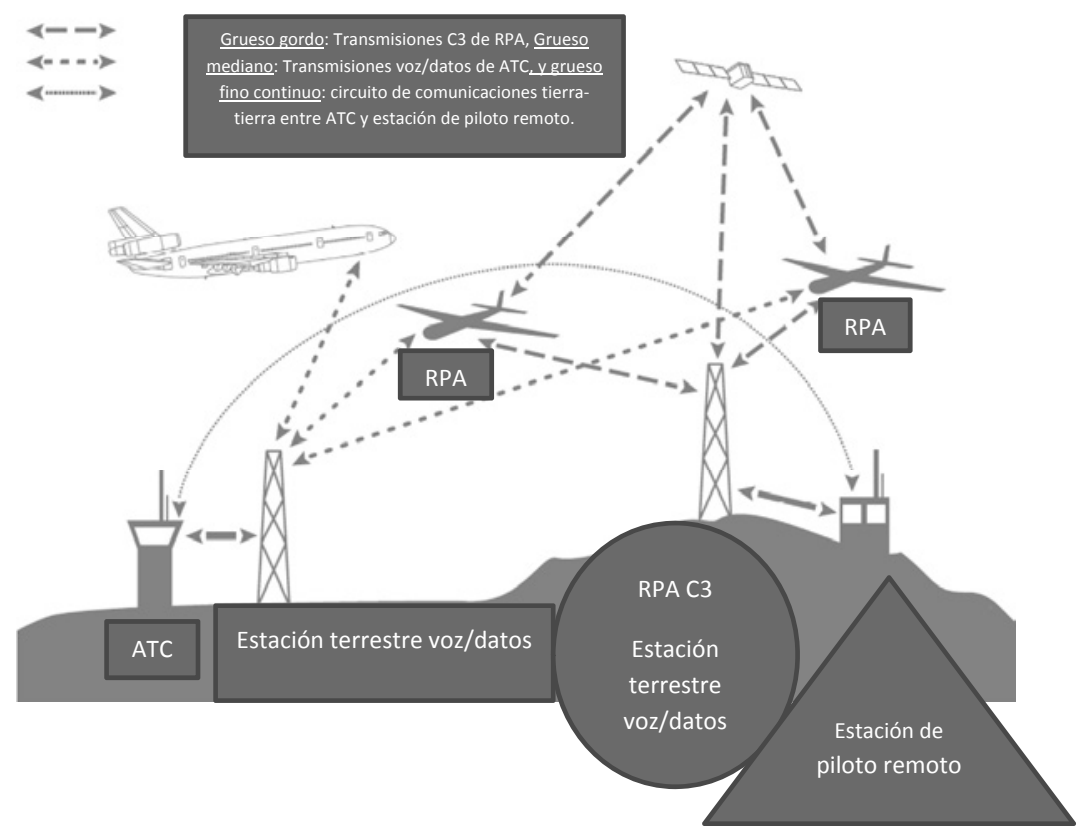




\section{Figura 4. Enlace de comunicaciones}

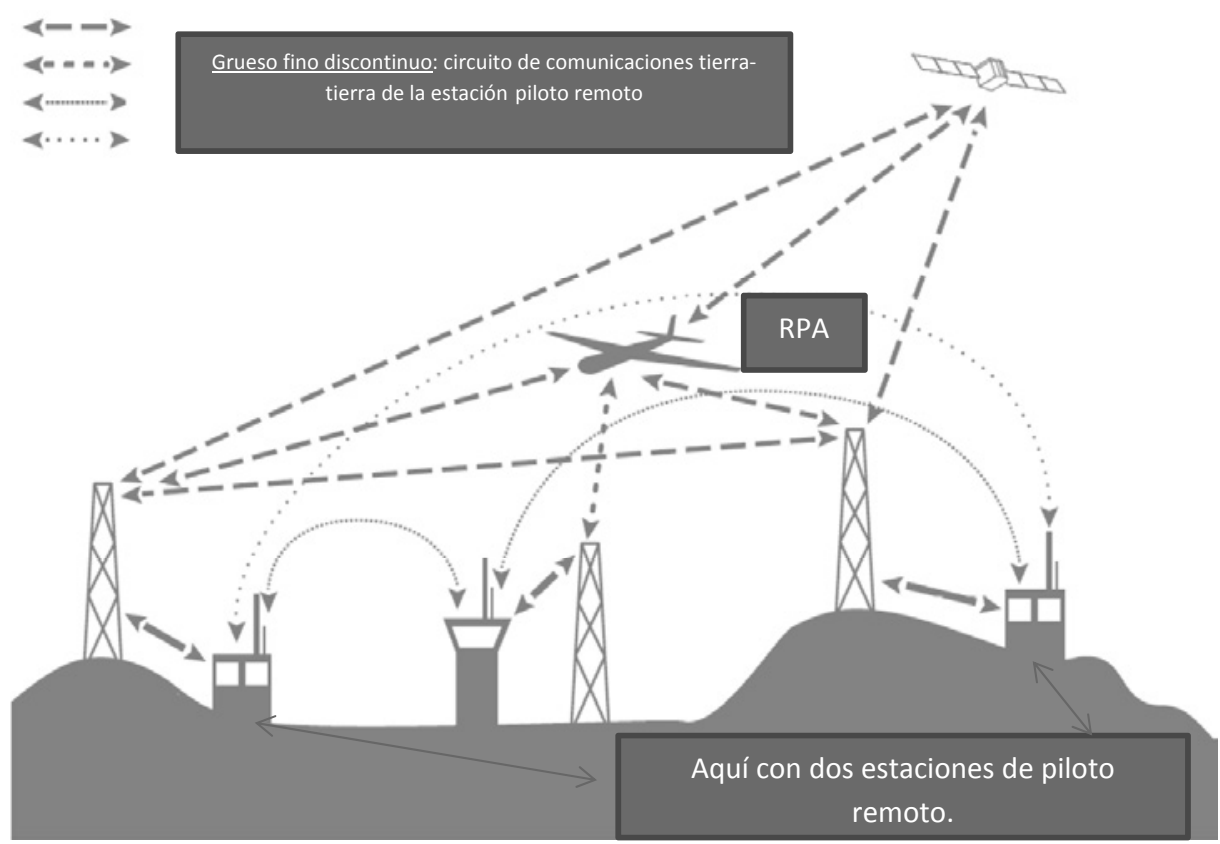


